\title{
ON SOLUTION OF FIRST ORDER INITIAL VALUE PROBLEMS USING LAPLACE TRANSFORM IN FUZZY ENVIRONMENT
}

\author{
Terrang, Abubakar Umar ${ }^{1 *}$, Isa, Awumtiya Kumba ${ }^{2}$, Felix Bakare ${ }^{3}$, Iliya, \\ Patience Bwanu ${ }^{1}$
}

1. Department of Mathematics, Federal University of Kashere, Gombe, Nigeria.

2. Department of Mathematics, Adamawa State College of Education, Hong.

3. Department of Mathematics, University of Lagos, Nigeria.

*Corresponding author: terrangabubakar@gmail.com

\section{Article Info}

Received: 13 January 2021

Accepted: 03 November 2021
Revised: 28 October 2021

Available online: 10 November 2021

\begin{abstract}
This study aimed at solving a nonhomogeneous linear first order initial value problem by means of Laplace transform method in fuzzy environment. The conditions for a fuzzy function to be $H$-differentiable and $g H$-differentiability are well established. Finally, example is constructed to test the applicability or otherwise of the established results.
\end{abstract}

Keywords: $H$-differentiable, $g H$-Differentiability, Fuzzy Initial Value Problem.

MSC2010: 03B52

\section{Introduction}

The study of fuzzy differential equation (FDE) has been extensively developed in recent years. FDE is considered as a proven important topic based on theoretical points of view. The idea of fuzzy number and fuzzy arithmetic were first introduced by Zadeh [1] and Dubois and Parade [2]. The term "Fuzzy Differential Equation" was conceptualized in 1978 by Kandel and Byatt [3] and right after two years, a larger version was published in [4]. The study of fuzzy differential in modelling hydraulic differential servo cylinders and fuzzy sets and systems were extensively discussed in [5] and [6] respectively. Fuzzy differential equations and initial value problem were extensively studied by other authors (see [7], [8] \& [9]). Also problems involving simulation of continuous fuzzy systems as well as linear non homogeneous ODE in fuzzy environment can be found in [10] and [11] respectively. Recently FDE has also used in many models such as HIV model [12], decay model [13], predator-prey and population models [14], civil engineering [15], modeling hydraulic. See also [16] and [17] for solutions of first order linear homogeneous ordinary differential equation by Laplace transform, a fish population problem in [18], an imprecise barometric pressure problem discussed in [19], an elementary application of population dynamics model in [20] and as well as arm race model considered in [21]. 
This paper presents a solution of nonhomogeneous first order linear fuzzy initial value problem by applying fuzzy Laplace transform method and established conditions for a fuzzy function to be H-differentiable and gH-differentiability respectively. Consider equation

$$
\frac{d x}{d t}=k x+x_{0}
$$

with initial condition $x\left(t_{0}\right)=\gamma$. Equation (1.1) is called fuzzy ordinary differential equation (FODE) if any one of the following three cases holds:

i Only $\gamma$ is a generalized fuzzy number (Type-I).

ii Only $k$ is a generalized fuzzy number (Type-II).

iii Both $k$ and $\gamma$ are generalized fuzzy numbers (Type-III).

Let the solution of equation (1.1) be $x(t)$ and its $\alpha$-cut be $x(t, \alpha)=\left[x_{1}(t, \alpha), x_{2}(t, \alpha)\right]$.

If $x_{1}(t, \alpha) \leq x_{2}(t, \alpha), \forall \alpha \in[0, \omega], 0<\omega \leq 1$, then $x(t)$ is called strong solution otherwise $x(t)$ is called weak solution and in that case $\alpha$-cut of the solution is given by

$$
x(t, \alpha)=\left[\min x_{1}(t, \alpha), x_{2}(t, \alpha), \max x_{1}(t, \alpha), x_{2}(t, \alpha)\right](\text { see } 1001[21])
$$

The $\alpha$-level or level of confidence at level $\alpha$ of fuzzy set $A$ of $X$ is a crisp set $A_{\alpha}$ that contains all the elements of $X$ that have membership values in $A$ greater than or equal to $\alpha$ i.e.

$$
A=\left(x, \mu_{A}(x)\right) \geq \alpha, x \in X, \alpha \in[0,1] .
$$

$A \in \tilde{F}$ is called a fuzzy number where $R$ denotes the set of whole real numbers if

i $\tilde{A}$ is normal i.e. $x_{0} \in R$ exists such that $\mu_{\tilde{A}}(x)=1$.

ii $\forall \alpha \in(0,1], A_{\alpha}$ is a closed interval.

If $A$ is a fuzzy number then $\tilde{A}$ is a convex fuzzy set and if $\mu_{\tilde{A}}(x)=1$ then $\mu_{\tilde{A}}(x)$ is non decreasing for $x \leq x_{0}$ and non-increasing for $x \geq x_{0}$. The membership function of a fuzzy number $\tilde{A}\left(a_{1}, a_{2}, a_{3}, a_{4}\right)$ is defined by

$$
\mu_{\tilde{A}}(x)= \begin{cases}1, & x \in\left[a_{1}, a_{2}\right] \neq \phi \\ L(x), & a_{1} \leq x \leq a_{2} \\ R(x), & a_{3} \leq x \leq a_{4}\end{cases}
$$

where $L(x)$ denotes an increasing function and $0<L(x) \leq 1, R(x)$ denotes a decreasing function and $0 \leq R(x) \leq 1$. A generalized fuzzy number is called a generalized triangular fuzzy number if it is defined by $\tilde{\tilde{A}}=\left(a_{1}, a_{2}, a_{3}, \omega\right)$ and its membership function is given by

$$
\mu_{\tilde{A}(x)}= \begin{cases}0, & x \leq a_{1} \\ \omega \frac{x-a_{1}}{a_{2}-a_{1}}, & a_{1} \leq x \leq a_{2} \\ \omega \frac{a_{3}-x}{a_{3}-a_{2}}, & a_{2} \leq x \leq a_{3} \\ 0, & x \geq a_{3}\end{cases}
$$


or $\mu_{\tilde{A}}(x)=\max \left(\min \left(\omega \frac{x-a_{1}}{a_{2}-a_{1}}, \omega, \quad \omega \frac{a_{3}-x}{a_{3}-a_{2}}\right), 0\right)$

A generalize trapezoidal fuzzy number is a subset of $R$ denoted as $\tilde{A}_{G T}=\left(a_{1}, a_{2}, a_{3}, a_{4} ; \omega\right)$ with the following membership function as follows

$$
\mu_{\tilde{A}}(x)= \begin{cases}\omega \frac{x-a_{1}}{a_{2}-a_{1}}, & a_{1} \leq x \leq a_{2} \\ \omega, & a_{2} \leq x \leq a_{3} \\ \omega \frac{a_{3}-x}{a_{3}-a_{2}}, & a_{3} \leq x \leq a_{4} \\ 0, & x \geq a_{4}\end{cases}
$$

To set conditions for fuzzy function to be $H$-differentiable and $g H$-differentiable respectively, let $f:(a, b) \longrightarrow R_{\tilde{F}}$ and $t_{0} \in(a, b)$, if $\exists f^{\prime}\left(t_{0}\right) \in R_{\tilde{F}}$ such that $\forall h>0$ sufficiently small, $\exists f^{\prime}\left(t_{0}\right)$ such that:

i $f^{\prime}\left(t_{0}\right)$ is H-differentiable, then $f^{\prime}\left(t_{0}\right)$ is called fuzzy derivative of $f(t)$.

ii If $f^{\prime}\left(t_{0}\right)$ is gH-differentiable, then $f^{\prime}\left(t_{0}\right)$ is called generalized fuzzy derivative of $f(t)$.

A function $f: T \longrightarrow E$ is said to be Hukuhara differentiable at $t_{0} \in T$, if there exists an element $f^{\prime}\left(t_{0}\right) \in E$ such that for all $h>0$ sufficiently small. In other words, there exists

$$
\begin{gathered}
f\left(t_{0}+h\right) \underline{H} f\left(t_{0}\right), f\left(t_{0}\right) \underline{H} f\left(t_{0}-h\right), f\left(t_{0}+h\right) \underline{H} f\left(t_{0}\right), f\left(t_{0}\right) \underline{H} f\left(t_{0}-h\right) \\
\lim _{h \rightarrow 0^{+}} \frac{f\left(t_{0}+h\right) \underline{H} f\left(t_{0}\right)}{h}=\lim _{h \rightarrow 0^{+}} \frac{f\left(t_{0}\right) \bar{H} f\left(t_{0}-h\right)}{h}=f^{\prime}\left(t_{0}\right)
\end{gathered}
$$

\section{Methodology}

To apply the Laplace transform method, we assume that the solution to equation (1.1) is piecewise continuous on a given interval $(a, b)$. In order to take the Laplace transform of equation (1.1), we need to obtain the transform of the derivative of the function. The idea of a transform is that, it turns a given function into another function. That is;

i The derivative $D$ takes a differentiable function $f$ (defined on some interval $(a, b)$ ) and assigned to it a new function $D f=f^{\prime}$.

ii The integral $I$ takes a continuous function $f$ (defined on some interval $[\mathrm{a}, \mathrm{b}]$ ) and assigned to it a new function $\operatorname{If}(x)=\int_{a}^{x} f(t) d t$.

iii The multiplication operator $M_{\phi}$, which multiplies any given function $f$ on the interval [a,b] by a fixed function $\phi$ on $[\mathrm{a}, \mathrm{b}]$, is a transform: $M_{\phi} f(x)=\phi(x) . f(x)$.

A key to the use of Laplace transform theory in differential equations is the way that $L$ treats derivatives. This means that

$$
L\left[y^{\prime}\right]=\int_{0}^{\infty} e^{-p x} y^{\prime}(x) d x
$$


In general,

$$
L\left[f^{n}(t)\right]=s^{n} f(s)-s^{n-1} f(0)-s^{n-2} f^{\prime}(0)-s^{n-3} f^{\prime \prime}(0)-s^{n-4} f^{\prime \prime \prime}(0)-\ldots-f^{n-1}(0)
$$

\section{Results}

This section presents the results we obtained for the generalized Hukahara differentiability by applying fuzzy Laplace transform method to a given equation. Therefore, consider a nonhomogeneous equation

$$
\frac{d x}{d t}=k x \pm t^{2}
$$

where $k$ is a constant with fuzzy initial condition $x\left(t_{o}\right)=\gamma$ as it also appeared in equation (1.1) above. Equation (3.1) is also called FODE if any one of the conditions (i) - (iii) for equation (1.1) holds.

Let $y^{\prime}(t)=\left(\underline{y}^{\prime}(t, \alpha), \bar{y}^{\prime}(t, \alpha)\right)$

$$
L\left[f\left(t, y(t), y^{\prime}(t)\right)\right]=\left\{p^{2} L\left[y(t)-p y_{o}\right]\right\}-z_{o}
$$

Hence

$$
\begin{aligned}
& L\left[\underline{f}\left(t, y(t), y^{\prime}(t), \alpha\right)\right]=p^{2} L[\underline{y}(t, \alpha)]-p \underline{y}_{o}(\alpha)-\underline{z}_{o}(\alpha) \\
& L\left[\bar{f}\left(t, y(t), y^{\prime}(t), \alpha\right)\right]=p^{2} L[\bar{y}(t, \alpha)]-p \bar{y}_{o}(\alpha)-\bar{z}_{o}(\alpha)
\end{aligned}
$$

where

$$
L\left[\underline{f}\left(t, y(t), y^{\prime}(t), \alpha\right)\right]=\min \left\{f(t, u, v), u \in(\underline{y}(t, \alpha), \bar{y}(t, \alpha)), v \in\left(\underline{y}^{\prime}(t, \alpha), \bar{y}^{\prime}(t, \alpha)\right)\right\}
$$

and

$$
L\left[\bar{f}\left(t, y(t), y^{\prime}(t), \alpha\right)\right]=\min \left\{f(t, u, v), u \in\left(\underline{y}(t, \alpha), \bar{y}^{\prime}(t, \alpha)\right), v \in\left(\underline{y}^{\prime}(t, \alpha), \bar{y}(t, \alpha)\right)\right\}
$$

Therefore, the solution of equation (3.4) is obtained and therefore, presented below.

$$
\begin{aligned}
& L[\underline{y}(t, \alpha)]=H_{1}(p, \alpha) \\
& L[\bar{y}(t, \alpha)]=K_{1}(p, \alpha)
\end{aligned}
$$

Taking the inverse Laplace transform of equation (3.5) and (3.6), the following are obtained

$$
\underline{y}(t, \alpha)=L^{-1}\left[H_{1}(p, \alpha)\right]
$$


and

$$
\bar{y}(t, \alpha)=L^{-1}\left[K_{1}(p, \alpha)\right]
$$

where $H_{1}(p, \alpha)$ and $K_{1}(p, \alpha)$ are solved uing the assumption that the fuzzy linear function $f$ is given by

$$
f\left(t, y(t), y^{\prime}(t)\right)=a y(t)+b y^{\prime}(t)+c(t),
$$

which is a crisp mapping and

$$
\begin{array}{r}
H_{1}(p, \alpha)=L[\underline{y}(t, \alpha)]=\frac{(p-b) \underline{y}_{0}(\alpha)+\underline{z}_{o}(\alpha)+L[c(t)]}{p^{2}-b p-a} \\
K_{1}(p, \alpha)=L[\bar{y}(t, \alpha)]=\frac{(p-b) \bar{y}_{o}(\alpha)+\underline{z}_{o}(\alpha)+L[c(t)]}{p^{2}-b p-a} \\
{\left[f^{\prime}(t)\right]_{\alpha}=\left[f_{2}^{\prime}\left(t_{o}, \alpha\right), f_{1}^{\prime}\left(t_{o}, \alpha\right)\right]} \\
L\left[f\left(t, y(t), y^{\prime}(t)\right)\right]=L\left[a y^{\prime}(t)+b y^{\prime \prime}(t)\right] \\
=a L\left[y^{\prime}(t)\right]+b L\left[y^{\prime \prime}(t)\right] \\
=a[s f(s)-f(0)]+b\left[s f(s)-s f(0)-f^{\prime}(0)\right] \\
=(a+b) s L[\underline{y}(t, \alpha)]-(a-b s) \underline{y}_{0}(\alpha)-b \underline{y}^{\prime}(\alpha)
\end{array}
$$

As a result of equations (3.2) and (3.3) we obtain,

$$
(a+b) p L[\underline{y}(t, \alpha)]-(a-b p) \underline{y}_{0}(\alpha)-b \underline{y}^{\prime}(\alpha)=p^{2} L[\underline{y}(t, \alpha)]-p y_{0}(\alpha)-\underline{z}_{0}(\alpha)
$$

solving equation (3.4) we arrive at

$$
(a+b) p L[\underline{y}(t, \alpha)]-p^{2} L[\underline{y}(t, \alpha)]=b \underline{y}^{\prime}(\alpha)+(a-b p) \underline{y}_{0}(\alpha)-p y_{0}(\alpha)-\underline{z}_{0}(\alpha)
$$

Rearranging equation (3.5) we have,

$$
L[\underline{y}(t, \alpha)]=\frac{b \underline{y}^{\prime}(\alpha)+(a-b p-p) \underline{y}_{0}(\alpha)-z_{0}(\alpha)}{a p+b p-p^{2}},
$$

which is

$$
(a+b) p L[\bar{y}(t, \alpha)]-(a-b) p \bar{y}_{0}(\alpha)-b \bar{y}^{\prime}(\alpha)=p^{2} L[\bar{y}(t, \alpha)]-p \bar{y}_{0}(\alpha)-\bar{z}_{0}(\alpha),
$$


Also we solve equation (3.7) to obtain

$$
L[\bar{y}(t, \alpha)]=\frac{b \bar{y}^{\prime}(\alpha)+(a-b p-p) \overline{y_{0}}(\alpha)-\overline{z_{0}}(\alpha)}{a p+b p-p^{2}}
$$

Therefore,

$$
\begin{gathered}
H_{a_{1}}(t, \alpha)=\frac{b \underline{y}^{\prime}(\alpha)+(a-b p-p) \underline{y}_{0}-z_{0}(\alpha)}{a p+b p-p^{2}} \\
K_{a_{1}}(t, \alpha)=\frac{b \bar{y}^{\prime}(\alpha)+(a-b p-p) \bar{y}_{0}(\alpha)-\bar{z}_{0}(\alpha)}{a p+b p-p^{2}}
\end{gathered}
$$

and finally,

$$
a p L[\underline{y}(t, \alpha)]+b p L[\underline{y}(t, \alpha)]-a \bar{y}_{0}(\alpha)+b p \bar{y}_{0}(\alpha)-b \bar{y}^{\prime}(\alpha)=p^{2} L[\underline{y}(t, \alpha)]-p \underline{y}_{0}(\alpha)-\underline{z}_{0}(\alpha)
$$

Rearranging equation (3.18) we have

$$
\begin{aligned}
(a+b) p L[\underline{y}(t, \alpha)]-p^{2} L[\underline{y}(t, \alpha)] & =b \bar{y}^{\prime}(\alpha)+a \bar{y}_{0}(\alpha)-b p \bar{y}_{0}(\alpha)-p \underline{y}_{0}(\alpha)-\underline{z}_{0}(\alpha), \\
\left(a p+b p-p^{2}\right) L[\underline{y}(t, \alpha)] & =(a-b p-p) \underline{y}_{0}(\alpha)+b \bar{y}_{0}^{\prime}(\alpha)-\underline{z}_{0}(\alpha) \\
L[\bar{y}(t, \alpha)] & =\frac{(a-b p-p) \underline{y}_{0}(\alpha)+b \bar{y}_{0}^{\prime}(\alpha)-\underline{z}_{0}(\alpha)}{a p+b p-p^{2}}
\end{aligned}
$$

Similarly,

$$
L[\underline{y}(t, \alpha)]=\frac{(a-b p-p) \bar{y}_{0}(\alpha)+b \bar{y}_{0}^{\prime}-\bar{z}_{0}(\alpha)}{a p+b p-p^{2}}
$$

Therefore,

$$
\begin{aligned}
& H_{b_{1}}(t, \alpha)=\frac{(a-b p-p) \underline{y}_{0}(\alpha)+b \bar{y}_{0}^{\prime}-\underline{z}_{0}(\alpha)}{a p+b p-p^{2}} \\
& K_{b_{1}}(t, \alpha)=\frac{(a-b p-p) \bar{y}_{0}(\alpha)+b \bar{y}_{0}^{\prime}-\bar{z}_{0}(\alpha)}{a p+b p-p^{2}}
\end{aligned}
$$

Based on what we obtained above therefore, an example is constructed below to test the applicability or otherwise the established results. Consider the first order FODE

$$
\frac{d x}{d t}=3 p^{2}+t
$$


where $p^{2}$ is a constant. To solve this type of equation we need to consider the following four cases as indicated in this paper.

Case I: Assume that if $f(x)$ and $f^{\prime}(x)$ are (i)-differentiable, then applying equation (3.23) to $f(x)$ and $f^{\prime}(x)$ respectively gives the following results to

$L\left[f^{\prime}(x)\right]=p L[f(x)](-) f(0)$ and

$L\left[f^{\prime \prime}(x)\right]=p L\left[f^{\prime}(x)\right](-) f^{\prime}(0)$.

Combining the two identities yields the result,

$$
\begin{aligned}
L\left[f^{\prime \prime}(x)\right] & =p\{p L[f(x)](-) f(0)\}(-) f^{\prime}(0) \\
& =\left\{p^{2} L[f(x)](-) p f(0)\right\}(-) f^{\prime}(0)
\end{aligned}
$$

Case II: Assume that $f(x)$ is (i)-differentiable and $f^{\prime}(x)$ is (ii)-differentiable, then applying equation (3.24) to $f(x)$ and $f^{\prime}(x)$ respectively results to

$L\left[f^{\prime}(x)\right]=p L[f(x)](-) f(0)$ and

$L\left[f^{\prime \prime}(x)\right]=\left(-f^{\prime}(0)\right)(-)(-p) L f^{\prime}(x)$.

Combining these identities yields the desired result,

$$
\begin{aligned}
L\left[f^{\prime \prime}(x)\right] & =\left(-f^{\prime}(0)\right)(-)(-p)\{p L[f(x)](-) f(0)\} \\
& =\left(-f^{\prime}(0)\right)(-)\left\{-p^{2} L[f(x)](-)(-p f(0))\right\}
\end{aligned}
$$

Case III: If $f(x)$ is (ii)-differentiable and $f^{\prime}(x)$ is (i)-differentiable, then applying equation (3.23) to $f(x)$ and $f^{\prime}(x)$ respectively we have,

$$
\begin{aligned}
& L\left[f^{\prime}(x)\right]=(-f(0))(-)(-p) L[f(x)] \text { and } \\
& L\left[f^{\prime \prime}(x)\right]=p L\left[f^{\prime}(x)\right](-) f^{\prime}(0)
\end{aligned}
$$

Again combining the two identities we obtain,

$$
\begin{aligned}
L\left[f^{\prime \prime}(x)\right] & =p\{(-f(0))(-)(-p) L[f(x)]\}(-) f^{\prime}(0) \\
& =-\left\{(-p f(0))(-)\left(-p^{2}\right) L[f(x)]\right\}(-) f^{\prime}(0)
\end{aligned}
$$


Lastly,

Case IV: If both $f(x)$ and $f^{\prime}(x)$ are (ii)-differentiable and then equation (3.23) \& (3.24) is applied to them respectively, the result obtained is presented thus;

$$
\begin{aligned}
L\left[f^{\prime}(x)\right] & =(-f(0))(-)(-p) L[f(x)] \text { and } \\
L\left[f^{\prime \prime}(x)\right] & =\left(-f^{\prime}(0)\right)(-)(-p) L\left[f^{\prime}(x)\right]
\end{aligned}
$$

Combining these identities we have,

$$
\begin{aligned}
L\left[f^{\prime \prime}(x)\right] & =\left(-f^{\prime}(0)\right)(-)(-p)\{(-f(0))(-)(-p) L[f(x)]\} \\
& =-\left(f^{\prime}(0)\right)(-)\left\{p f(0)(-) p^{2} L[f(x)]\right\}
\end{aligned}
$$

\section{Conclusion}

It is of importance here to state that the example constructed and solved based on the results we established in this work has further justified our claims and the possibilities of solving linear nonhomogeneous first order ODE in fuzzy environment by means of fuzzy Laplace transform method. All the conditions considered and referred to are well satisfied and the results obtained are valid for the solution of generalized fuzzy initial valued problem of linear nonhomogeneous type.

\section{Declarations of interest}

The authors declare that there is no competing interest, neither was there any funding received for this research.

\section{Acknowledgement}

We wish to express our gratitude to the reviewers of this article, their observations and suggestions have greatly influenced the final stage of the work. Their constructive comment has helped improve the readability and preciseness of the manuscript.

Furthermore, we appreciate the discussions with Dr. A. Tahir of the Department of Mathematics, Modibbo Adama University, Yola, for his mentorship. Finally, our appreciations go to Prof Olaleru of the Department of Mathematics and Computer Science, University of Lagos, and the Editorial Board for their kind suggestions and patience.

\section{References}

[1] L. A. Zadeh, Fuzzy sets, Information and Control, 8(1965), 338-353.

[2] Dubois, D and H.Parade 1978, Operation on Fuzzy Number. International Journal of Fuzzy system, 9:613-626.

[3] A. Kandel and W. J. Byatt, "Fuzzy differential equations," in Proceedings of the International Conference on Cybernetics and Society, pp. 1213-1216, Tokyo, Japan, 1978. 
[4] A. Kandel and W. J. Byatt, "Fuzzy processes," Fuzzy Sets and Systems, vol. 4, no. 2, pp. $117-152,1980$.

[5] A. Bencsik, B. Bede, J. Tar, J. Fodor, Fuzzy differential equations in modeling hydraulic differential servo cylinders, in: Third Romanian_Hungarian Joint Symposium on Applied Computational Intelligence, SACI, Timisoara, Romania, 2006.

[6] J. J. Buckley and T. Feuring, "Fuzzy differential equations," Fuzzy Sets and Systems, vol. 110, no. 1, pp.43-54, 2000.

[7] B. Bede, I.J. Rudas, A.L. Bencsik, First order linear fuzzy differential equations under generalized differentiability, Information Sciences 177 (2007) 1648-1662.

[8] James J. Buckley, Thomas Feuring, Fuzzy initial value problem for Nth-order linear differential equations, Fuzzy Sets and Systems 121 (2001) 247-255.

[9] J.J. Buckley, T. Feuring, Y. Hayashi, Linear System of first order ordinary differential equations: fuzzy initial condition, soft computing 6 (2002) 415-421.

[10] L.J. Jowers, J.J. Buckley, K.D. Reilly, Simulating continuous fuzzy systems, Information Sciences 177 (2007) 436-448.

[11] S. R. Mondal, T. K. Roy, first order linear non homogeneous ordinary differential equation in fuzzy environment. Journal of Mathematica Theory and Modelling, 3 (1), (2013).

[12] Hassan Zarei, Ali Vahidian Kamyad, and Ali Akbar Heydari, Fuzzy Modeling and Control of HIV Infection, Computational and Mathematical Methods in Medicine Volume 2012, Article ID 893474, 17 pages.

[13] G.L. Diniz, J.F.R. Fernandes, J.F.C.A. Meyer, L.C. Barros, A fuzzy Cauchy problem modeling the decay of the biochemical oxygen demand in water, 2001 IEEE.

[14] L.C. Barros, R.C. Bassanezi, P.A. Tonelli, Fuzzy modelling in population dynamics, Ecol. Model. 128 (2000) 27-33.

[15] M. Oberguggenberger, S. Pittschmann, Differential equations with fuzzy parameters, Math. Modelling Syst. 5 (1999) 181-202.

[16] S. R. Mondal, T. K. Roy, first order linear homogeneous ordinary differential equation in fuzzy environment based on laplace transform. Journal of mathematics computer science. 3 (2013) $1533-1564$.

[17] S. R. Mondal, T. K. Roy, first order linear non homogeneous ordinary differential equation in fuzzy environment based on laplace transform. Journal fuzzy set valued analysis. (2013) 1-18.

[18] S. R. Mondal, T. K. Roy, first order linear homogeneous ordinary differential equation in fuzzy environment based on lagranges multiplier method. Journal of Uncertainty in Mathematical Science, 3 vol. 2014 (2014) 1-17.

[19] S. R. Mondal, T. K. Roy, first order linear homogeneous ordinary differential equation in fuzzy environment. International Journal of Pure and Applied Sciences and Technology, 14(1) (2013) $16-26$.

[20] S. R. Mondal, T. K. Roy, solution of second order linear ordinary differential equation in fuzzy environment. Journal of Fuzzy Mathematics and Informatics, 3 (2015) 1-31.

[21] S. R. Mondal, T. K. Roy, system of differential equation with initial value as triangular intuitionistic fuzzy number and its application. International Journal of Computer Mathematics, 3 (2015) 449-474. 